\title{
A Hybrid Technique for De-Noising Multi-Modality Medical Images by Employing Cuckoo's Search with Curvelet Transform
}

\author{
QAISAR JAVAID*,MUHAMMAD ARIF**, MUNAM ALI SHAH***, AND MUHAMMAD NADEEM* \\ RECEIVED ON 20.06.2016 ACCEPTED ON 22.11.2016
}

\begin{abstract}
De-noising of the medical images is very difficult task. To improve the overall visual representation we need to apply a contrast enhancement techniques, this representation provide the physicians and clinicians a good and recovered diagnosis results. Various de-noising and contrast enhancements methods are develops. However, some of the methods are not good in providing the better results with accuracy and efficiency. In our paper we de-noise and enhance the medical images without any loss of information. We uses the curvelet transform in combination with ridglet transform along with CS (Cuckoo Search) algorithm. The curvlet transform adapt and represents the sparse pixel informations with all edges. The edges play very important role in understanding of the images. Curvlet transform computes the edges very efficiently where the wavelets are failed. We used the CS to optimize the de-noising coefficients without loss of structural and morphological information. Our designed method would be accurate and efficient in de-noising the medical images. Our method attempts to remove the multiplicative and additive noises. Our proposed method is proved to be an efficient and reliable in removing all kind of noises from the medical images. Result indicates that our proposed approach is better than other approaches in removing impulse, Gaussian, and speckle noises.
\end{abstract}

Key Words: Curvelet Transform, Medical Images, Noise, Cuckoo Search, De-Noising, Optimization.

\section{INTRODUCTION}

$\mathrm{T}$ he primary objective of images in our real life is to understand a huge amount of data in a prompt view rather than browsing and understanding a large number of papers. Therefore, images have a good form of information to perceive something. Medical images make it possible for an expert to analyze and detect diseases. Images may be distorted because of dissimilar types of noises during acquisition and communication and therefore require enhancement and de-noising. Denoising of images is necessary to extract useful information through medical images. Image processing methods can be utilized to enhance, reconstruct, and analyze an image or its areas of interest for users.Two noise models exist, namely, multiplicative and additive [1]. Additive or multiplicative noise can cause a corrupted image. In salt and pepper noises, pixels, which are corrupted, have either

Corresponding Author (E-Mail: qaisar@iiu.edu.pk)

* Department of Computer Science \& Software Engineering, International Islamic University, Islamabad.

** Department of Computer Science, University of Gujrat, Gujrat.

*** Department of Computer Science, COMSATS Institute of Information Technology, Islamabad.

Mehran University Research Journal of Engineering \& Technology, Volume 37, No. 1, January, 2018 [p-ISSN: 0254-7821, e-ISSN: 2413-7219] 
very low or high intensities in comparison with intensity of neighboring pixel. A pixel in an image is considered as salt pixel when it contains high abnormal values. Salt and pepper noises acquire values of either 0 or 255, i.e. the random values of noise can vary between 0 and 255 . Noise can be added to an image through multiplicative rule. A multiplicative noise model comprises speckle and Rician noises. Such type of noises is considered as data missing noises and arises when loss of data occurs during signal transmission. Imaging systems related to medical, such as MRI (Magnetic Resonance Imaging) and CT imagery can be often corrupted by speckle noise. Speckle noise follows the gamma distribution. Curvelet with ridgelet transform approaches would be our main research focus. For comparison, we use wavelet-based methods, i.e. Sure shrink [2], Visu shrink [3], Bayes shrink [4], and NNs (Neural Networks) [5]. Noises on medical images are found to be Rician, Gaussian, and speckle.

\section{RELATED WORK}

MRI, ultrasound, and CT images are useful to diagnose diseases in the medical field. High-quality images present good diagnosis results. Therefore, making such type of noise-free images is important. In this study, we only focus on CT and MIRs. These types of images are suffering from impulse, speckle, Rician, and Gaussian noises [6]. Pizurica [7] suggested a method for de-noising magnetic resonance and ultrasound images. They exploited wavelets to remove speckle and Rician noises. Probability density function and empirically approximating probabilities are the basics for this method. The suggested method is not complex and is useful for unidentified types of noises.

Another useful method was suggested in [8]; the concept of dyadic wavelets was utilized, with soft thresholding of expansion coefficient thresholding for 3D (Three Dimensional) X-Ray images. A method for de-noising MRI with white Gaussian noise was mentioned in [9]. Bilateral filter was then used to approximate coefficients by denoising and preserving edges. The de-noised coefficient was used for the formation of reconstructed images. A technique for preserving edges of magnetic resonance images while removing noises was highlighted in [10].

Images related to tumor of breast cancer were de-noised by thresholding NNs in [11]. Two steps were performed for this method. First, the image was de-noised to become clear. Second, image segmentation was performed to extracts the region of interests. Thresholding NNs were combines with wavelets to obtain de-noising and efficient detection of medical images.

However, considerable time is required for a machine to show intelligent behavior. Thus, such types of techniques are time-consuming. Research related to medical images has highlighted the use of two AI techniques, i.e. fuzzy set theoryand NNs. De-noising can be effectively performed using both types of softcomputing techniques. The learning capability of ANN (Artificial Neural Network) and parallel computing are useful for several applications, i.e. computer vision, pattern recognition, signal processing, and imaging. However, such capability presents several drawbacks, such as lack of generality, dependence on many parameters, and cannot represent knowledge. Separate architecture of ANN is required for the problem of specificity, even for minute changes related to noise dataset and structure. During the training of ANN, the parameters that are to be determined are the learning rate, neurons in each layer, input weights, number of hidden layers, and transfer function. With the use of ANN, knowledge representing explicitly also becomes an issue. Meanwhile, fuzzy logic systems cannot handle numerical data. If large numbers of patterns exist in a dataset, then using if-then rule for fuzzy logic systems becomes difficult for extraction [12].

Mehran University Research Journal of Engineering \& Technology, Volume 37, No. 1, January, 2018 [p-ISSN: 0254-7821, e-ISSN: 2413-7219] 
For smoothing an image, a fuzzy filter was suggested in [12.13]. Efficient results for de-noising could be produced with the preservation of image details. However, this filter wasted much time performing de-noise without noise detection. The suggested technique in [14] used NN for images performing principal component analysis. Owing to limitations for $\mathrm{NN}$, this technique had some disadvantages. Stopping criteria for the learning process of NN also affected this technique. The technique suggested in [11] was de-noising medical images using a wavelet technique thresholded with NN. A new threshold strategy has been proposed by this technique for wavelets and trains the $\mathrm{NN}$ for such type of problem. Satisfactory results have been achieved for wavelets using this strategy.

Image smoothing can be performed using wavelets by eliminating components of some high frequency, which may be contaminated with some noise. During wavelet shrinkage methods, threshold value can be computed. Differentiation between noisy wavelet coefficients and the coefficients having useful information can be conducted using thresholding [15]. Coefficients of wavelets lower than the threshold are set to 0 , whereas the remaining coefficients are altered or maintained with respect to thresholding strategy. Selection of threshold is critical for algorithms based on wavelet shrinkage. Good de-noising results are difficult to obtain using inaccurate values for threshold.

An image can be filtered with the moving of window mask upon the image. To implement and to design a filter are simple. However, they are not much effective because of deficiency of knowledge about image structures and noise types. Another reason for low accuracy rate is the selection for appropriate size of window. On one hand, texture or detail from noise cannot be distinguished using small sizes of windows. On the other hand, large sizes of windows may lead to false detection for noise with the covering of new texture, and the image may be blurred.
Pragada and Sivaswamy [16], the suggested technique compared fixed bio-orthogonal wavelets with matched wavelets using Bi shrink and bays strategies for thresholding for different images. The suggested method showed an improvement for the values of low PSNR (Peak Signal-to-Noise Ratio) noisy images that ranged from 018. Hence, high values for noise can be removed using this method. To de-noise images, the techniques highlighted in $[17,18]$ applied complex wavelet transform to images. The technique in [19] focused on preserving shift invariance for medical and satellite images and details for fine image. To achieve such goals, this method was successful with the use of complex wavelets. Bayesian estimator was used in this technique. In the technique presented in [17], a new estimator was designed using Stein's principle.

Bayesian estimator and ridglet transform was used for the techniques suggested in $[15,20,21]$ to recover components of signal from coefficients of wavelets. Compared with other models, Bayesian estimator can be utilized to obtain accurate approximation of impulse noise. A difference exists between the two techniques, i.e. the first approach is useful for poison noise, whereas the second and third approach is useful for both poison and Gaussian noises.

Speckle noise frequently corrupts SAR (Synthetic Aperture Radar) images. The authors in [22] used an ICA (Independent Component Analysis). Results showed that ICA performed better than Lee and Kuan filter. Another discrete curvelet transform-based method [23] was proposed to de-noise typhoon cloud images. Gaussian noise from a typhoon cloud image was removed by combining generalized cross validation and discrete curvelet transform. The proposed methodology outperformed wavelets using generalized cross validation and soft threshold methods. 
Rashedi and Zarezadeh [24], a filtering technique was applied on medical images to remove Gaussian noise. The authors proposed three new filters, and their performance was better than other nonlinear filters. The shortcoming of nonlinear filters is their tendency toward image blurring. Qing-Hang [25] also discussed a filtering method based oncombinations of anisotropic diffusion filters and linear minimum mean square error filters. Techniques proposed in $[9,26]$ were based on the merger of contrast enhancement and de-noising methods.

Alternately, the probability distribution function of wavelet coefficients that gives useful information was determined by unknown noise types in [27], and the Canny edge detector [25] was used after thresholding to enhance edges. The wavelet coefficients, which are helpful in Rician and speckle noise reduction from medical images, were approximated using bilateral filter $[26,28]$. Hence, the result satisfaction was based on these techniques.Wavelets [29] and simple curvelet [30] are not reliant on noise type. They are applied to remove all types of noises, but their performance is not much satisfactory in terms of accuracy, morphological and structural information, and computational complexity. The quality of images can be improved further by developing more efficient techniques than NNs and wavelets.

\section{PROPOSED METHODOLOGY}

In the given section, we explain the curvelet transform by using ridgelet transforms with CS for medical image denoising. Fig. 1 shows the block diagrams of the proposed method, which consist of some essential stages.

(1) The frequency of thecurvelet coefficients of the input images are de-noised by employingCS, which combines the output of the images in terms of objective evaluations, such as PSNR, MSE, SSIM, DSSIM, CNR, and UIQI.
(2) The frequency of the curvelet coefficients of the images are selected from the source images.

(3) The inverse curvelet transform is applied for frequencycurvelet coefficients to generate the final smooth image.

(4) Curvelet transform is applied for the image decompositions, partitioning, analysis of the component, and renormalizations.

(5) The function of curvelet transform is modified according to CS.

(6) Every image square is analyzed by DRTs (Discrete Ridgelet Transforms).

(7) A dimensionals featuresvectors are extracted fromimages by employing thecurvelet transform.

(8) CS is used to optimize the feature for image smoothing.

\subsection{Curvelet Transform}

The frequency and time analysis decomposes a signal into the numerouso rthogonals bases. The signals are quantizes into the summations of the different coefficient basis, i.e. $\mathrm{f}=\Sigma \mathrm{k}$ ak bk whre ak is the coefficients, and bk is the basis, frame. Although wavelet can efficiently handle point discontinuity [31,32], but curvelet consider numerous coefficients for edges, as shown in Fig. 2. Fig. 2(a) shows that the wavelet approach requires many wavelet coefficients to interpret edges, singularities along lines, or curves. Fig. 2(b) shows that the curvelet approach needs minimal coefficient for edges. There are mainly two types of curvelet transforms, first and second generation of curvelet transforms. The first generation curvelet transform has complicated digital realization that includes sub-band division, smoothing block, normalization, and ridgelet analysis. The ridgelet analysis is so complicated 
to implement and takes too much time for analysis.The second generation curvelet transform is also known as fast curvelet transform, and it reduces the computational time for ridgelet analysis. It uses wrapping algorithms such as continuous, discrete ridgelet transform and RT (Radon Transform). Overviews of curvelet and ridgelet transforms are given in Fig. 3.

The overview of curvelet transform is given in Fig. 3.
Fig. 3 illustrates the decompositions of input images intothe sub-bands by using spatials partitionings of every sub-bands (every sub-band decomposes into blocks). The ridgelet transforms is then used to every blocks. We describe the bank of sub-band filter $\mathrm{P}_{0},\left(\Delta_{s}, \mathrm{~s} \geq 0\right)$. The entities $\mathrm{f}$ is filters into the sub-band. This stage divided the images into several resolutions layer. Each layer includes detail of diverse frequency, as explained in the following Equation (1):

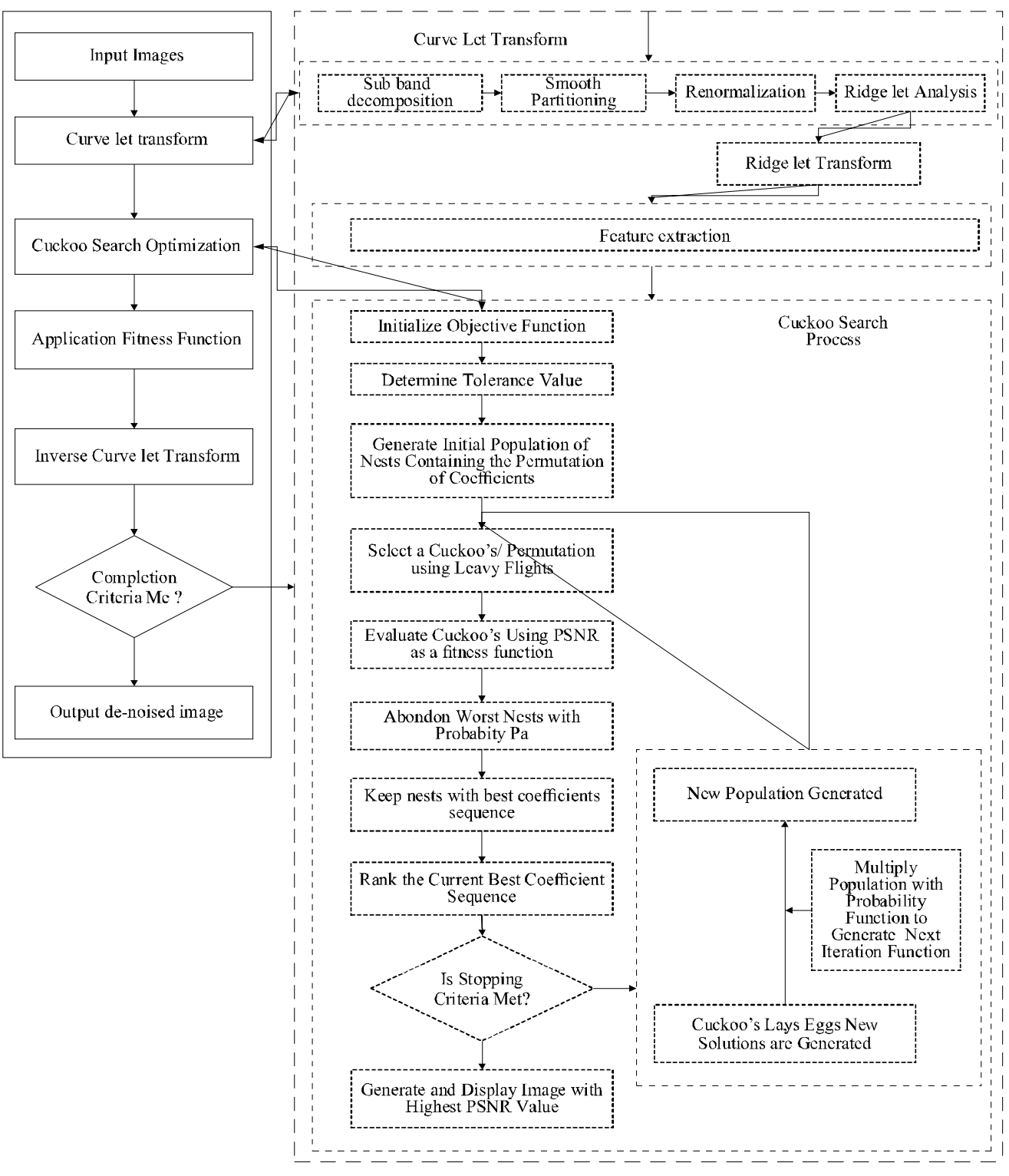

FIG. 1. THE FLOW MODEL OF THE PROPOSED WORK

Mehran University Research Journal of Engineering \& Technology, Volume 37, No. 1, January, 2018 [p-ISSN: 0254-7821, e-ISSN: 2413-7219] 
$f^{\prime} !\left(P_{0} f, D_{1} f, D_{2} f, \ldots.\right)$

Where $\mathrm{P}_{0}$ and $\Delta_{1} \cdot \Delta_{2}, \ldots$ are the high and low frequencies filters. Therefore, the input images can be restructured from the sub-bands through Equation (2).

$f=P_{0}\left(P_{0} f\right)+\sum_{s} \Delta_{S}\left(\Delta_{s} f\right)$

A convolution operator is applied for sub-band decomposition, as explained in Equation (3).

$\mathrm{P}_{0}, \mathrm{f}=\mathrm{F}_{0} * \mathrm{f} \mathrm{D}_{\mathrm{s}} \mathrm{f}=\mathrm{Y}_{2 \mathrm{~s}} * \mathrm{f}$

Some connections exist among curvelets and wavelets transform. The sub-band decomposition can be estimated by using the familiar wavelet transforms. The wavelet transforms are decomposed into different decomposition levels, such as $\mathrm{S}_{0}, \mathrm{D}_{1}, \mathrm{D}_{2}, \mathrm{D}_{3} . \mathrm{P}_{0} \mathrm{f}$ is moderately developed from $\mathrm{S}_{0}$ to $\mathrm{D}_{1}$ and may comprise $\mathrm{D}_{2}$ and $\mathrm{D}_{3} . \Delta_{\mathrm{s}} \mathrm{f}$ is assembled from $\mathrm{D}_{2 \mathrm{~s}}$ to $\mathrm{D}_{2 \mathrm{~s}+1}$. $\mathrm{P}_{0} \mathrm{f}$ is low pass and can be capably represented using wavelet base [33]. However, the discontinuity curve affects the high-pass layers $\Delta \mathrm{s}$ f. A compilation of smooth window $\mathrm{WQ}\left(\mathrm{x}_{1}, \mathrm{x}_{2}\right)$ localize about dyadic image squares is defined.

The next step is to smooth the partitioning of the image defined as a compilation of the smooth windows, and the ridglet analysis and ridglet transform, The used of the approach in association with the ridgelet transform has been deliberated in [33-35].

Fig. 4(a-c) illustrates an institutive example of magnetic resonance image by using wavelet and curvelet transforms. Input image can be decomposed into more high-pass sub-bands than only three sub-bands of the wavelet transform. Therefore, curvelet transform with ridgelet transform captures more feature information in different directional sub-bands than wavelet transform does.

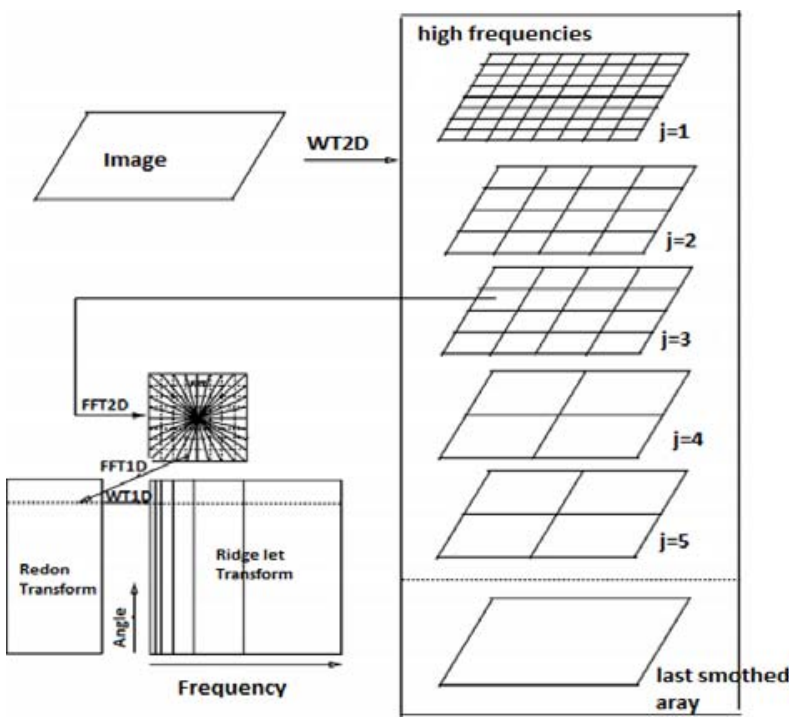

FIG. 3.CURVELET TRANSFORM FLOW GRAPH

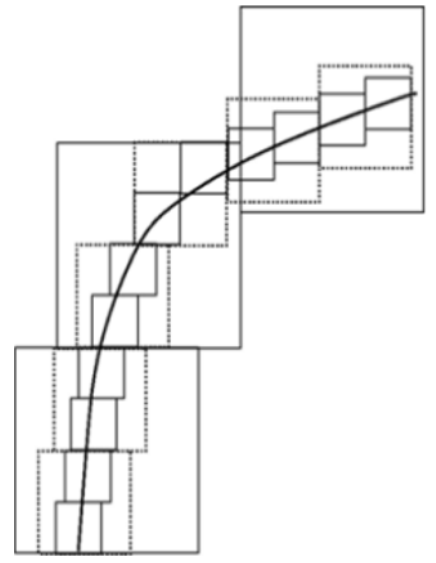

FIG. 2(a). WAVELET TRANSFORM

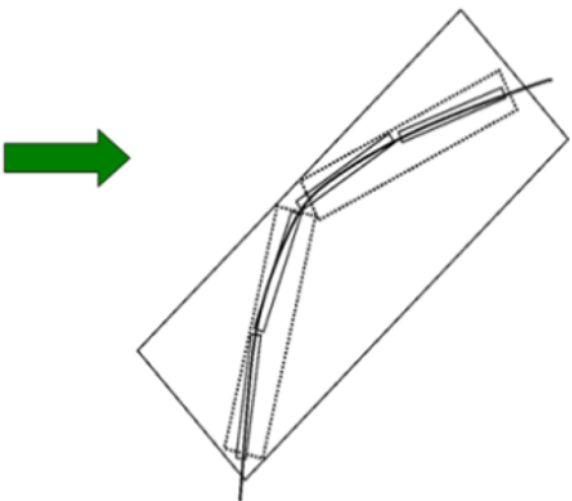

FIG. 2(b). CURVELET TRANFORM

Mehran University Research Journal of Engineering \& Technology, Volume 37, No. 1, January, 2018 [p-ISSN: 0254-7821, e-ISSN: 2413-7219] 
Fig. 5 Illustrate the transformation of digital polar grid in frequencies domains for nxn matrix image where $n$ is equal to length 8. Curvelet transform flowgraph. Fig. 5 illustrates the decomposition of the original image into subbands followed by the spatial partitioning of each sub-band.The ridgelet transform is then applied to each block.

\subsection{De-Noising Coefficients}

After decomposing the images into sub-bands and applying the ridglet and RT, we employing our technique to de-noise the medical image. The proposed methodology is ordinary and is outlined mostly for selfcontentedness and clearness.

Noisy data are given in the form of the following Equation (4):

$x_{i, j}=f(i, j)+s z_{i, j}$

where $\mathrm{f}$ is source input image to be de-noised and enhanced, and $z$ is the noise, i.e. $z_{i, j}{ }^{\text {i.i.d. }} \mathrm{N}(0,1)$ added to the image and $\sigma$ is used to summation the total noise in the image.

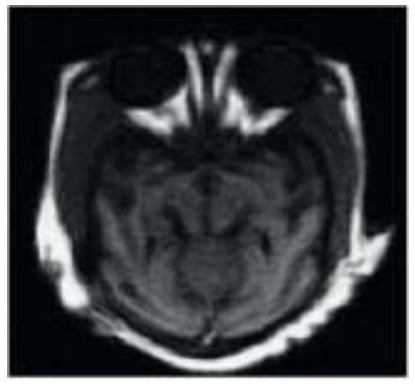

FIG. 4(a). SOURCE IMAGE

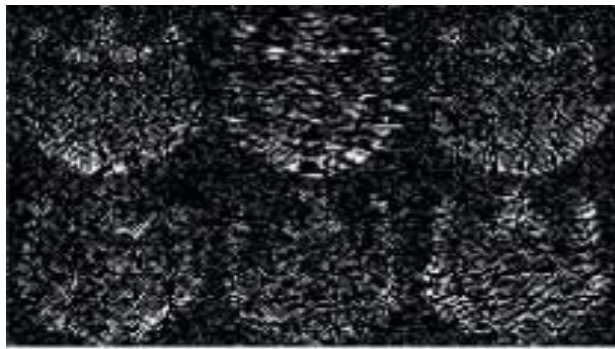

FIG. 4(d). SIX HIGH-PASS SUB-BANDS IN THE $2^{N D}$ LEVEL

\subsection{Cuckoo Search}

In the given section we introduces CS algorithm to optimizethe de-noised curvelet coefficients. In our method we combine the curvelet abridglet transform with an optimization technique named as CS. The main function of CS is to optimize and maintain the main feature and morphological structure of an image without loss of

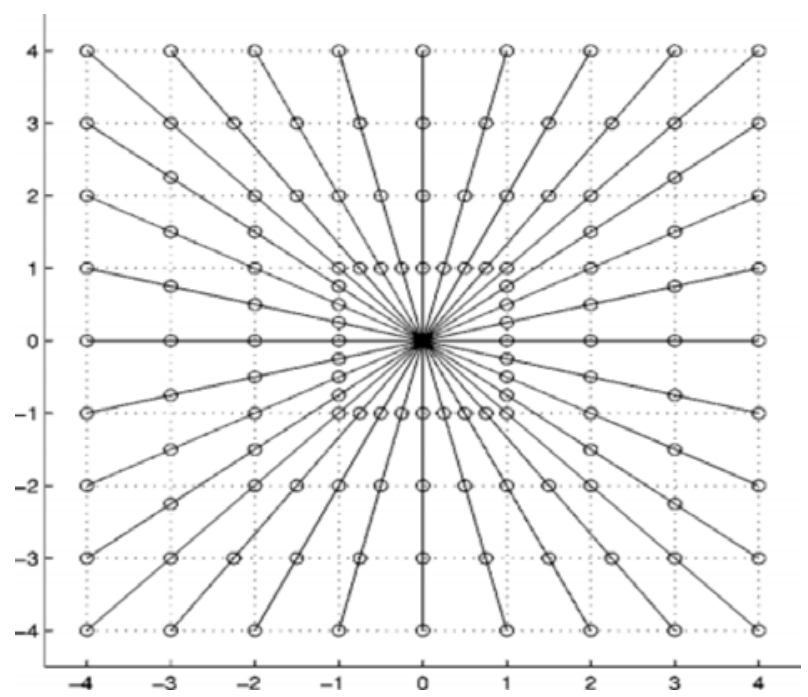

FIG. 5. THE ILLUSTRATIONS OF DIGITAL POLAR GRID IN FREQUENCIES DOMAINS FOR $N X N$ IMAGE $(N=8)$

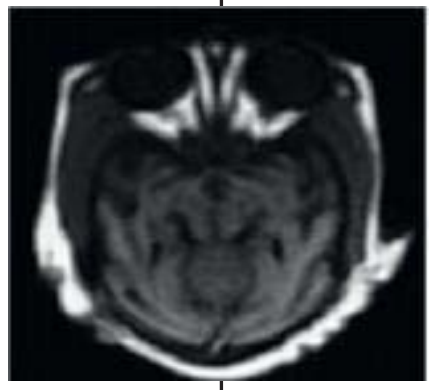

FIG. 4(b). LOW-PASS SUB-BANDS

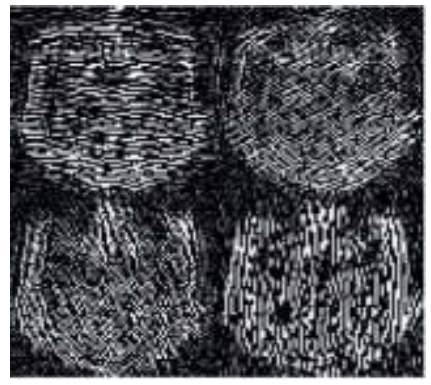

FIG. 4(c). FOUR HIGH-PASS SUBBANDS IN THE FIRST LEVEL

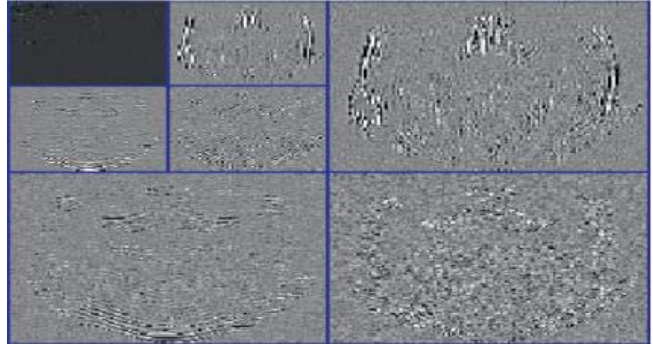

FIG. 4(e). AN EXAMPLE OF THE WAVELET TRANSFORM

Mehran University Research Journal of Engineering \& Technology, Volume 37, No. 1, January, 2018 [p-ISSN: 0254-7821, e-ISSN: 2413-7219] 
information. The curvelet transform has its own characteristics in removing noise from the medical images at different intensities and variances.

The CS techniques is a nature inspired algorithm, and it attracts the so much attention from the researchers. The Cuckoo Birds lays their eggs in the other birds nests, and then they select and remove the nests intelligently, to increase the probability of the eggs hatching. The host birds either throw away the eggs or migrates to the other nests. The random walks of the cukoo helps to selects the similar nests. Levy distributions used infinites means and variances to presents the randoms steps lengths of levy flights, as given in Equation (5) [36]. In Equation (5) $\mu$ indicate the Levy distribution and $\lambda$ indicate the random walk length. The Levy flight essentially provide random steps length is drawn from the levy distribution.

Levy $\sim \mathrm{m}=\mathrm{t}^{-1},(1<\mathrm{l} \leq 3)$

CS employs breedings and the flyings behaviors of the cuckoo. The basic CS behavior are given below [36].

- $\quad$ Population contains nests with eggs.

- $\quad$ Eggs represented problems and solution. The Cuckoo egg is considered as the new solution.

- If the cuckooegg match to the eggs of the hosts, then the finding probability is limited. The solutions ofthefitness function is ondifferent invariance of the solution.

The CS algorithm has its roots in three rules given below [36].

- $\quad$ Every cuckoo can lay dump randomly one egg at the time in the selected nest.

- $\quad$ Next generations will inherit the best solution.

- $\quad$ The eggs of the host nests remains unaffected, and the probability of egg discovery by a host egg is pa of $[0,1]$.
Eggs represent a solution. Only the most excellent solutions are conceded to subsequent generation to attain the goal rapidly. Solution is evaluated by fitness function [37].

According to [36], several advantages of CS algorithm are presented below:

- $\quad$ CS ensures that the local optimum problem is not occurring because most of the solutions are generated by randomization, with locations beyond the best solutions.

- $\quad$ CS randomization is more efficient than PSO and GA

- $\quad$ CS is more general than GA and PSO because it needs a minimal number of parameters given that CS is adopted in optimization problems.

- $\quad$ CS algorithm is used to determine the sequence of de-noised coefficients, in which PSNR and other objective evaluation parameters are used as fitness function.

Random permutations of the curvelet transform coefficients are generated. A nest includes one permutations as an egg. The egg or permutations size are fixed to four in our case. Therefore, every permutations contains four randoms coefficients. The initials populations of the nestin fact includes permutations of smoothing the coefficients. Fitness is recognizedby the computings the objective evaluation parameters. Only the best nests are approved for the next generation based on the objective evaluation parameter values. New solutions are produced by conducting random walks.

\subsection{Inverse Curvelet Transform}

After the de-noising through the curvelet coefficients, we apply inverse curvelet transform to reconstruct image. Inversing the procedure of curvelet transforms with some

Mehran University Research Journal of Engineering \& Technology, Volume 37, No. 1, January, 2018 [p-ISSN: 0254-7821, e-ISSN: 2413-7219] 
mathematic revising ridgelet synthesis means that each image square is restructured from the orthonormal ridgelet classification. All ridgelet coefficients are summed with basis, as described in Equation (6), where $\mathrm{g}$ is the ridglet coefficients, $\alpha$ and pare the length and density of the coefficients.

$g_{Q}=\sum_{\lambda} \alpha_{(Q, \lambda)} \cdot \rho_{\lambda}$

Renormalization means that each image square resulting in preceding stages is renormalized to its own appropriate image squares, as described in Equation (7), Where Q is the smooth combination, $h$ indicates the stages of the images.

$h_{Q}=T_{Q} g_{Q}$

Where $\mathrm{Q} \in \mathrm{Q}$ Smooth combinations means that we inverse the windows analysis to all the windows restructurings in the precedings stage of image, $w$ indicates the sub bands window, as described in Equation (8).

$$
\Delta_{s} f=\sum_{Q \in Q_{s}} w_{Q} \cdot h_{Q}
$$

In sub-bands re-compositions, we reverse bank of subband filter using the replicate Equation (9) to sum all the sub-bands, where $\mathrm{P}_{0}$ are the sub band filters.

$$
f=P_{0}\left(P_{0} f\right)+\sum_{s} \Delta_{s}\left(\Delta_{s} f\right)
$$

\section{EXPERIMENTAL SETUP}

In this his section we elaborates and discuss the experimental procedure for image de-noising using curvelet and CS. To verify the proposed de-noising scheme, we do experiments over several varieties of source images including MRI and CT. The experimental platform is MATLAB 2012a. The computer clocked is 3.40 core i7 and the computer memory is $8 \mathrm{~GB}$. Valuation of image denoising and enhancement algorithms is challenging. The process frequently includes the quality, computational cost, and universality of the algorithm. Many quantitative measures can be used to evaluate the quality of de-noised images. As discussed in the literature, PSNR is a mathematical method used to measure image quality. PSNR is exploited to assess results produced by different denoising algorithms.

To check the performance of the proposed approach, some standard CT and MRI scan images are selected for testing. We select a dataset of 100 images with different resolutions to evaluate the proposed technique. Resolutions are 256x256, 512x512, and 1024x1024.

\subsection{Objective Evaluation}

To evaluates the performance of a variety of a methods, quality assessments methods, such as PSNR, CNR, UIQI, SSIM, and distance SSIM (DSSIM), are applied on images, and the parameters included in this research are shown in Table 1.

\subsubsection{PSNR}

The PSNR is defined as follows in Equation (10) [38]:

TABLE 1. PARAMETERS USED IN IMPLEMENTATION

\begin{tabular}{|c|c|}
\hline Parameters & Values \\
\hline Image type & Gray-scale medical image \\
\hline Noise types & Gaussian, impulse, and speckle \\
\hline Range of Gaussian noise & $0.01-0.2$ \\
\hline Range of impulse noise & $0.01-0.5$ \\
\hline Range of speckle noise & $0.01-0.1$ \\
\hline Wavelet family & Duberchies \\
\hline Decomposition level & 2 \\
\hline Gamma value & $2-4$ \\
\hline Sigma value & $10-30$ \\
\hline Initial population & 4 \\
\hline Probability of finding bad nests & 0.25 \\
\hline Step size & 0.05 \\
\hline
\end{tabular}

Mehran University Research Journal of Engineering \& Technology, Volume 37, No. 1, January, 2018 [p-ISSN: 0254-7821, e-ISSN: 2413-7219] 
$P S N R=20 \log _{10} \frac{255}{\sqrt{M S E}}$

Where MSE is equal to:

$M S E=\frac{1}{\alpha \times \beta} \sum_{i=0}^{\alpha-1} \sum_{j=0}^{\beta-1}[0(i, j)-c(i, j)]^{2}$

When medical images are acquired by any means, it refers to the unnecessary turbulence that induces into the image.

\subsubsection{SSIM and DSSIM}

SSIM method is used to measure similarity between two images. This method can be considered as a quality measure for one of the images being compared by fulfilling the condition that the second image has a perfect quality [39].

The SSIM metric is calculated as follows in Equation (11):

$\operatorname{SSIM}(x, y)=\frac{\left(2 \mu_{x} \mu_{y}+c 1\right)\left(2 \sigma_{x y}+c 2\right)}{\left(\mu_{x}^{2}+\mu_{y}^{2}+c 1\right)\left(\sigma_{x}^{2}+\sigma_{y}^{2}+c 2\right)}$

$\operatorname{DSSIM}(x, y)=\frac{1-\operatorname{SSIMM}(x, y)}{2}$

\subsubsection{CNR}

CNR is described in Equation (13).

$C N R_{A B}=\left(S N R_{A}-S N R_{B}\right)=\left(\frac{\left(S_{A}-S_{B}\right)}{\sigma_{0}}\right)$

$\mathrm{SNR}=\mathrm{S}_{\mathrm{A}} / \sigma_{0} \mathrm{CNR}$ is the CNR between images $\mathrm{A}$ and B. $\sigma_{0}$ presents the noise in the images. The noise is assumed to be same for everyone. $\mathrm{M}$ and $\mathrm{N}$ are the sizes of the images. $\mathrm{S}_{\mathrm{A}}$ and $\mathrm{S}_{\mathrm{B}}$ are the intensities of the images.

\subsubsection{UIQI}

When image quality is being measured, a reliable technique that considers numerous features in measurements is used. Therefore, UIQI is used because it considers three features, such as contrast, luminance, and structural similarities, in determining the quality between the source and thede-noised images. The result is in the range of $[1,-1]$; if the values are near to one, then they are considered the best values.

The evaluation formula [40] is described as follows in Equation (14):

$U I Q I=\frac{4 \mu_{X} \mu_{Y} \sigma_{X Y}}{\left(\mu_{X}^{2}+\mu_{Y}^{2}\right)\left(\sigma_{X}^{2}+\sigma_{Y}^{2}\right)}$

\section{RESULTS AND ANALYSIS}

In this section, images are demonstrated to confirms the achievement of the proposed technique. We apply our proposed method on some degraded images and the results is presented in Fig.6. The left column in Fig. 6 is the degraded images and on the right column is the enhanced and de-noised image using the proposed methods.The images Fig.6(a) and Fig.6(c)are degraded images and the images Fig.6(b) and Fig.6(d) are enhanced images.

Our proposed method outperform the other methods in removing theRician, Gaussians, impulse and speckles noises. For the impulse noises at variances of 0.01 and 0.05, the Visu, Sure, and Bayes shrink methods perform efficiently, but they could not perform good on high variance values with high-resolution images. Our proposed work shows acceptable PSNR values at 0.01 and 0.05 variances, which are very near to the Visu, Sure, and Bayes shrink method results. Therefore, the proposed method is appropriate for removing impulse noise with high levels of noise. The proposed technique efficiently removed the all types of noises at low and high intensities at different variances. The de-noising behavior of the proposed method makes it more dependable than other methods. Tables 2-3 shows the behavior of the proposed approach at several intensities of impulse noise on different CT scan images. 


\subsection{Single-Bleeding Pattern CT Image}

Single-bleeding pattern CT and magnetic resonance images are used to analysis the behaviors of the proposed algorithm on multiplicative and additive noise models.

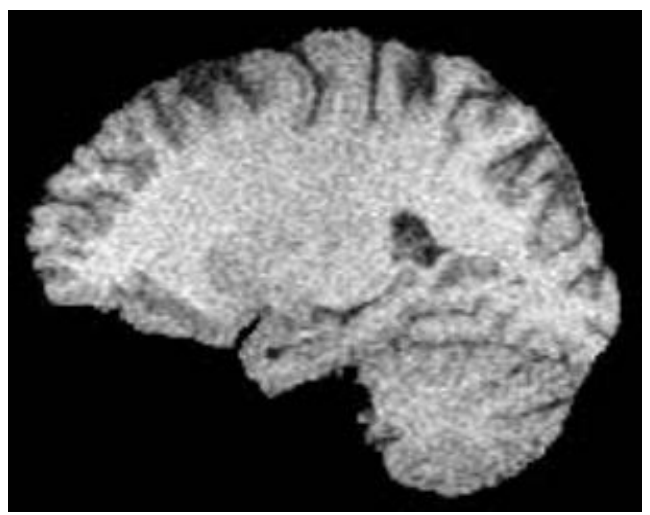

(a) SOURCE IMAGE

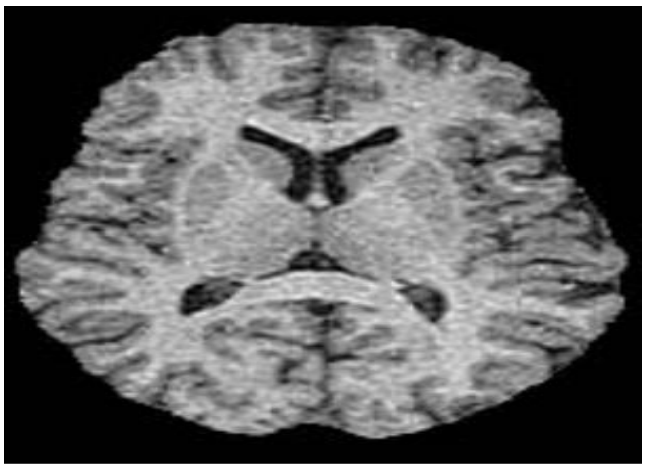

(c) ORIGINAL IMAGE
Table 2 shows the PSNR values of de-noised images obtained after applying various de-noising techniques. The proposed method is accurate. Figs. 7-8 Shows the PSNR value after adding Gaussian noise with a variance of 0.01 .

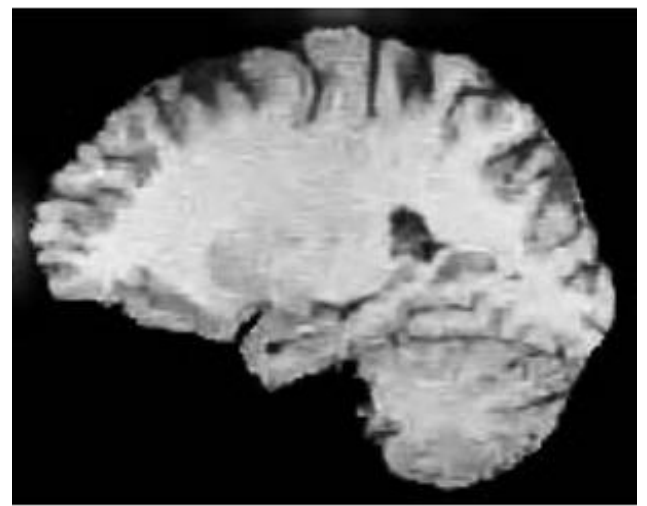

(b) ENHANCED AND DE-NOISED IMAGE

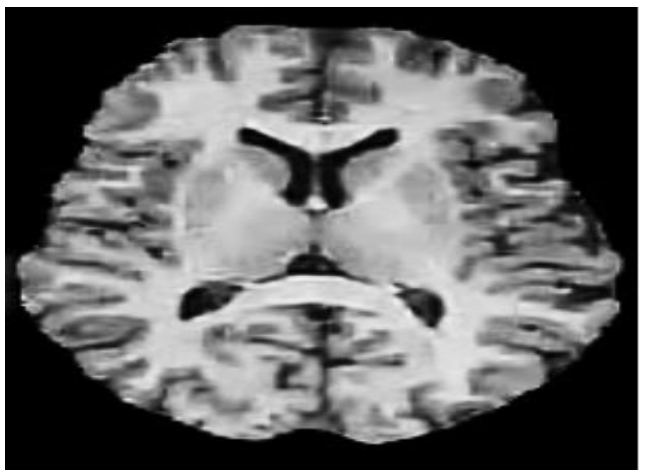

(d) ENHANCED AND DE-NOISED IMAGE

FIG. 6. IMAGES ON THE LEFT COLUMN ARE DEGRADED MAGNETIC RESONANCE IMAGES, AND THOSE ON THE RIGHT COLUMN ARE THE ENHANCED AND DE-NOISED IMAGES USING THE PROPOSED METHODS

TABLE 2. PSNR MEASURED VALUES AFTER ADDING GAUSSIAN NOISE AT DIFFERENT VARIANCE VALUES

\begin{tabular}{|c|c|c|c|c|c|c|c|}
\hline \multirow{2}{*}{ Different Resolutions Images } & \multicolumn{5}{|c|}{ Variance 0.01} & \multicolumn{5}{|c|}{ Variance 0.1} \\
\cline { 2 - 8 } & \multicolumn{7}{|c|}{ Image Matrix Size } \\
\cline { 2 - 8 } & $256 \times 256$ & $512 \times 512$ & $1024 \times 1024$ & $256 \times 256$ & \multicolumn{2}{|c|}{$512 \times 512$} & $1024 \times 1024$ \\
\hline Compared Techniques & \multicolumn{7}{|c|}{ Values } \\
\hline DTCWPT & 20.24 & 25.74 & 25.74 & 18.78 & 23.70 & 23.61 \\
\hline Visu shrink & 19.88 & 22.42 & 19.33 & 16.60 & 18.49 & 13.29 \\
\hline Bayes shrink & 19.98 & 22.43 & 19.28 & 16.58 & 18.48 & 13.29 \\
\hline Sure shrink & 15.04 & 15.30 & 15.45 & 13.93 & 14.92 & 11.21 \\
\hline BMWT & 16.30 & 16.75 & 16.99 & 14.10 & 15.20 & 15.70 \\
\hline AMC-SSDA & 17.23 & 18.20 & 18.45 & 17.10 & 17.12 & 18.10 \\
\hline Proposed Sequence & 25.37 & 32.22 & 32.22 & 23.17 & 29.31 & 29.31 \\
\hline
\end{tabular}

Mehran University Research Journal of Engineering \& Technology, Volume 37, No. 1, January, 2018 [p-ISSN: 0254-7821, e-ISSN: 2413-7219] 


\subsection{Multi-Bleeding Patterns}

Multi-bleeding pattern CT and MRI are used to analysis the behaviors of the proposed algorithm on multiplicative and additive noise models. Tables 4-6 shows the PSNR values of de-noised images obtained after applying various de-noising techniques. The proposed method is accurate and efficient in Figs. 9-10shows the PSNR value after adding Gaussian noise with a variance of 0.01 . Tables
7-9 shows the different PSNR values of de-noised images obtained after applying various de-noising techniques and get the speckle, impulse, rician and salt and paper noise values.

The preceding tables indicate that PSNR value increases as resolution increases at low noise intensities. At 0.5 variance, PSNR value decreases with an increase in the resolution. Our proposed method performs efficiently at

TABLE 3. PSNR MEASURED VALUES AFTER ADDING SPECKLE NOISE AT DIFFERENT VARIANCE VALUES

\begin{tabular}{|c|c|c|c|c|c|c|c|}
\hline \multirow{2}{*}{ Different Resolutions Images } & \multicolumn{5}{|c|}{ Variance .01 } & \multicolumn{5}{c|}{ Variance 0.5} \\
\cline { 2 - 8 } & \multicolumn{7}{|c|}{ Image Matrix Size } \\
\cline { 2 - 8 } & $256 \times 256$ & $512 \times 512$ & $1024 \times 1024$ & $256 \times 256$ & \multicolumn{2}{|c|}{$512 \times 512$} & $1024 \times 1024$ \\
\hline Compared Techniques & \multicolumn{7}{|c|}{ Values } \\
\hline DTCWPT & 23.93 & 30.72 & 30.72 & 20.24 & 25.70 & 25.74 \\
\hline Visu shrink & 22.66 & 24.80 & 25.07 & 19.23 & 17.45 & 21.50 \\
\hline Bayes shrink & 21.38 & 22.50 & 20.25 & 18.34 & 19.34 & 16.22 \\
\hline Sure shrink & 19.38 & 17.50 & 22.25 & 17.32 & 15.34 & 19.35 \\
\hline BMWT & 21.10 & 20.19 & 20.70 & 20.98 & 19.10 & 1.23 \\
\hline AMC-SSDA & 22.19 & 21.86 & 21.67 & 19.10 & 19.20 & 19.70 \\
\hline Proposed Sequence & 31.45 & 38.96 & 38.54 & 27.36 & 34.70 & 34.70 \\
\hline
\end{tabular}
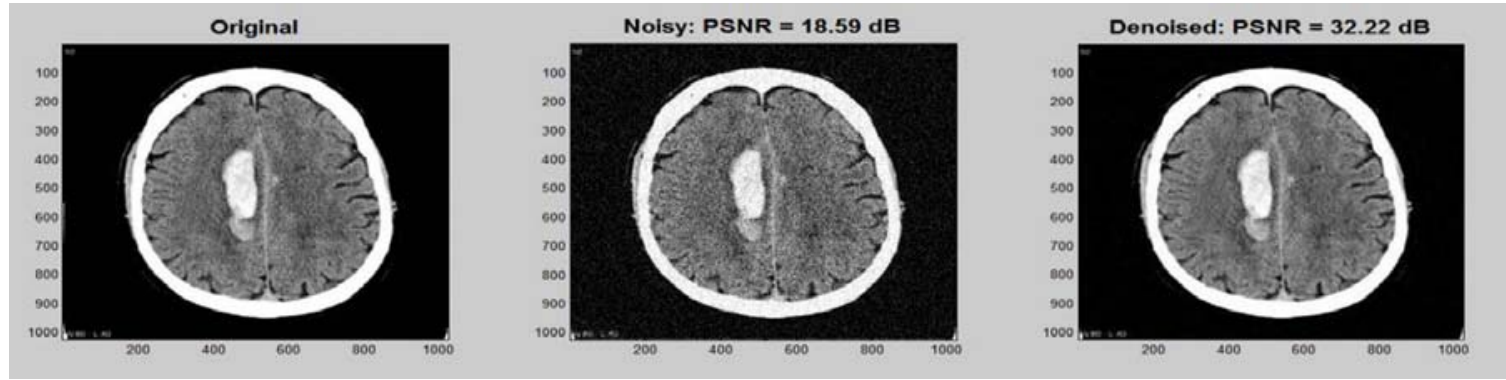

FIG. 7. PSNR VALUE AFTER ADDING GAUSSIAN NOISE WITH A VARIANCE OF 0.01
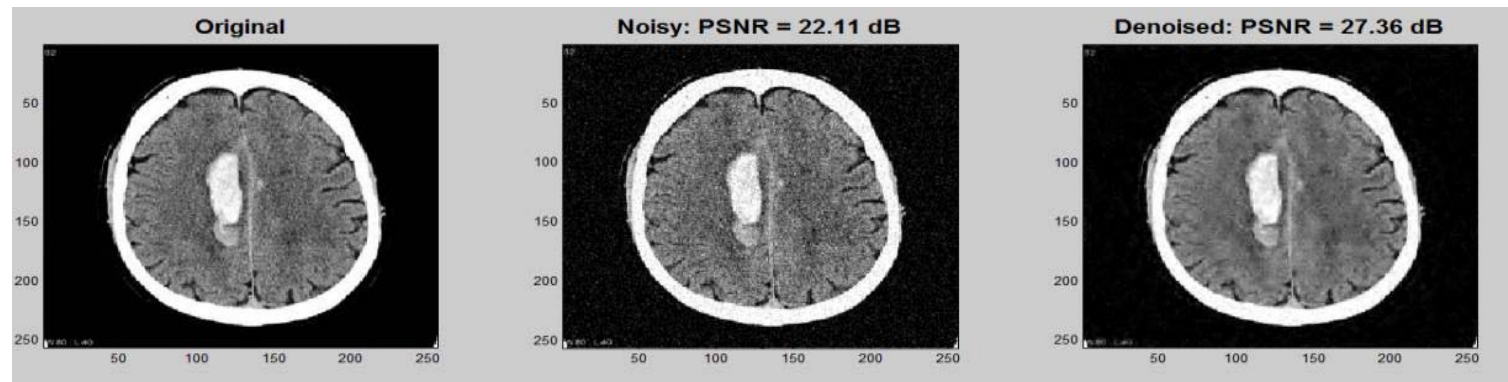

FIG. 8. PSNR VALUE AFTER ADDING SPECKLE NOISE WITH A VARIANCE OF 0.01

Mehran University Research Journal of Engineering \& Technology, Volume 37, No. 1, January, 2018 [p-ISSN: 0254-7821, e-ISSN: 2413-7219] 


\section{A Hybrid Technique for De-Noising Multi-Modality Medical Images by Employing Cuckoo's Search with Curvelet Transform}

0.5 variance with high-resolution images. Tables 8-9 present the behavior of the proposed approach for Gaussian and speckle noises at different noise intensities, respectively.
We illustrates the performances of the proposed technique on an MRI images and compare it to the traditional wavelets and some other methods. In Figs. 11-12(a) is the reference image effected with noise. In Figs. 11-12(a)

TABLE 4. PSNR MEASURED VALUES AFTER ADDING GAUSSIAN, IMPULSE, AND SPECKLE NOISES AT DIFFERENT VARIANCE VALUES

\begin{tabular}{|c|c|c|c|c|c|c|c|c|c|}
\hline \multirow{3}{*}{ Different Resolution Images } & \multicolumn{3}{|c|}{ Variance 0.1} & \multicolumn{3}{|c|}{ Variance 0.1} & \multicolumn{3}{|c|}{ Variance 0.1} \\
\hline & \multicolumn{9}{|c|}{ Image Matrix Size } \\
\hline & 256 x256 & $512 \times 512$ & $1024 \times 1024$ & $256 \times 256$ & 512 x512 & $1024 \times 1024$ & $256 \times 256$ & $512 \times 512$ & $1024 \times 024$ \\
\hline Compared techniques & \multicolumn{9}{|c|}{ Values } \\
\hline DTCWPT & 19.87 & 20.96 & 20.47 & 26.25 & 29.83 & 33.08 & 25.54 & 29.19 & 32.33 \\
\hline Visu shrink & 18.32 & 19.12 & 20.21 & 14.95 & 18.96 & 16.30 & 21.06 & 23.46 & 25.88 \\
\hline Bayes shrink & 18.00 & 18.24 & 19.49 & 13.99 & 17.45 & 16.45 & 20.15 & 23.46 & 25.00 \\
\hline Sure shrink & 15.30 & 15.01 & 15.15 & 12.37 & 15.34 & 13.53 & 17.38 & 19.39 & 20.55 \\
\hline BMWT & 22.10 & 22.70 & 22.10 & 21.19 & 21.13 & 21.09 & 22.19 & 22.90 & 22.99 \\
\hline AMC-SSDA & 24.15 & 24.67 & 24.80 & 22.10 & 22.90 & 23.01 & 22.91 & 22.96 & 23.10 \\
\hline Proposed Sequence & 26.50 & 29.94 & 33.50 & 27.17 & 30.84 & 34.34 & 24.49 & 28.20 & 31.85 \\
\hline
\end{tabular}

TABLE 5. PSNR MEASURED VALUES AFTER ADDING RICIAN NOISE

\begin{tabular}{|c|c|c|c|c|c|c|c|c|c|}
\hline \multirow{3}{*}{ Different Resolution Images } & \multicolumn{3}{|c|}{ Variance 0.01} & \multicolumn{3}{|c|}{ Variance 0.05} & \multicolumn{3}{|c|}{ Variance 0.1} \\
\hline & \multicolumn{9}{|c|}{ Image Matrix Size } \\
\hline & 256 x256 & $512 \times 512$ & $1024 \times 1024$ & 256 x256 & $512 \times 512$ & $1024 \times 1024$ & $256 \times 256$ & $512 \times 512$ & $1024 \times 024$ \\
\hline Compared techniques & \multicolumn{9}{|c|}{ Values } \\
\hline DTCWPT & 29.38 & 35.28 & 36.34 & 26.83 & 31.87 & 33.73 & 27.76 & 30.32 & 33.62 \\
\hline Visu shrink & 28.23 & 29.96 & 30.16 & 24.83 & 24.58 & 25.65 & 22.24 & 23.79 & 19.31 \\
\hline Bayes shrink & 29.71 & 30.06 & 30.56 & 25.86 & 24.23 & 24.54 & 23.97 & 24.80 & 25.20 \\
\hline Sure shrink & 24.33 & 25.02 & 26.72 & 18.10 & 17.49 & 19.27 & 18.86 & 19.12 & 20.45 \\
\hline BMWT & 27.10 & 27.70 & 27.10 & 26.19 & 26.13 & 26.09 & 25.19 & 25.90 & 25.99 \\
\hline AMC-SSDA & 25.15 & 25.67 & 25.80 & 26.10 & 26.90 & 27.01 & 28.91 & 28.96 & 28.10 \\
\hline Proposed Sequence & 32.96 & 34.54 & 37.99 & 30.45 & 33.60 & 36.51 & 26.43 & 29.08 & 32.61 \\
\hline
\end{tabular}

TABLE 6. PSNR MEASURED VALUES AFTER ADDING IMPULSE NOISE AT DIFFERENT VARIANCE VALUES

\begin{tabular}{|c|c|c|c|c|c|c|c|}
\hline \multirow{2}{*}{ Different Resolution Images } & \multicolumn{5}{|c|}{ Variance 0.01} & \multicolumn{5}{|c|}{ Variance 0.05} \\
\cline { 2 - 7 } & \multicolumn{7}{|c|}{ Image Matrix Size } \\
\cline { 2 - 7 } & $256 \times 256$ & $512 \times 512$ & $1024 \times 1024$ & $256 \times 256$ & $512 \times 512$ & $1024 \times 1024$ \\
\hline Compared techniques & \multicolumn{7}{|c|}{ Values } \\
\hline DTCWPT & 26.54 & 30.19 & 33.49 & 27.01 & 30.13 & 33.41 \\
\hline Visu shrink & 29.77 & 30.65 & 27.85 & 27.77 & 26.00 & 21.26 \\
\hline Bayes shrink & 31.02 & 29.34 & 26.25 & 24.23 & 22.75 & 19.37 \\
\hline Sure shrink & 29.22 & 28.94 & 26.21 & 24.47 & 23.77 & 20.80 \\
\hline BMWT & 18.30 & 18.75 & 18.99 & 17.10 & 17.20 & 17.70 \\
\hline AMC-SSDA & 19.23 & 19.20 & 19.45 & 18.10 & 18.12 & 18.10 \\
\hline Proposed Sequence & 27.30 & 30.96 & 34.46 & 27.23 & 30.90 & 34.34 \\
\hline
\end{tabular}

Mehran University Research Journal of Engineering \& Technology, Volume 37, No. 1, January, 2018 [p-ISSN: 0254-7821, e-ISSN: 2413-7219] 
Original magnetic resonance image, Fig. 12(b) image denoised with DTCWPT, Fig. 12(c) image de-noised with Visu shrink, Fig. 12(d) image de-noised with Bayes shrink, Fig. 12(e) image de-noised with Sure shrink Fig. 12(f) image de-noised with BMWT, Fig. 12(g) image de-noised with AMC-SSDA Fig. 12(h) image de-noised with the proposed methods. The de-noising results of the proposed technique clearly outperforms the result of the other approaches. The quantitative performance of the methods, for the Figs. 11-12 are shown in Table 10. The proposed approach performs better de-noising than other algorithms do for different kinds of images, such as singlebleeding patterns, multi-bleeding pattern images, and other general medical images.

Compared with traditional methods, our proposed method shows better performance in terms of accuracy. The resulted images show that the traditional methods cannot preserve the visual and morphological contents of the image and degrade the brightness of the image. Our
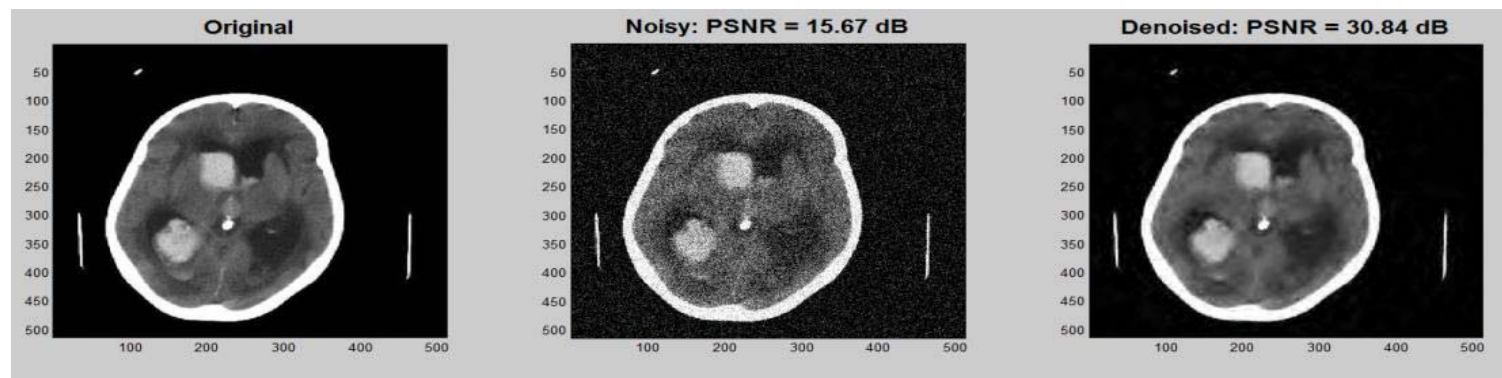

FIG. 9. AFTER ADDING IMPULSE NOISE AT A VARIANCE OF 0.1
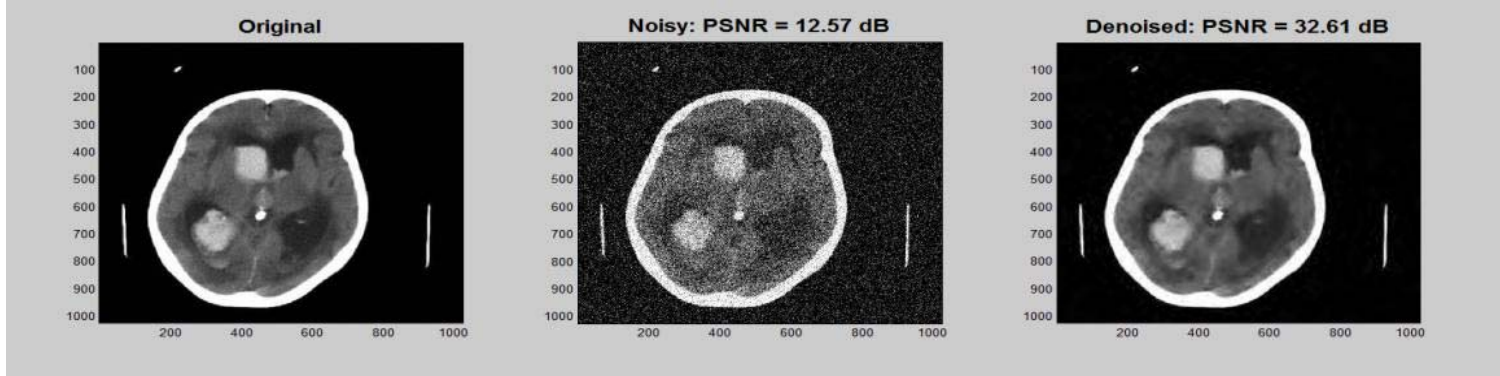

FIG. 10. PSNR VALUE AFTER ADDING GAUSSIAN NOISE WITH A VARIANCE OF 0.1

TABLE 7. PSNR MEASURED VALUES AFTER ADDING IMPULSE NOISE AT DIFFERENT VARIANCE VALUES

\begin{tabular}{|c|c|c|c|c|c|c|}
\hline \multirow{3}{*}{ Different Resolution Images } & \multicolumn{3}{|c|}{ Variance 0.3} & \multicolumn{3}{|c|}{ Variance 0.5} \\
\hline & \multicolumn{6}{|c|}{ Image Matrix Size } \\
\hline & $256 \times 256$ & $512 \times 512$ & $1024 \times 1024$ & $256 \times 256$ & $512 \times 512$ & $1024 \times 1024$ \\
\hline Compared techniques & \multicolumn{6}{|c|}{ Values } \\
\hline DTCWPT & 26.25 & 29.83 & 33.08 & 26.01 & 29.63 & 32.81 \\
\hline Visu shrink & 26.42 & 23.87 & 18.84 & 21.15 & 18.81 & 13.36 \\
\hline Bayes shrink & 21.82 & 20.96 & 17.45 & 21.70 & 19.34 & 14.35 \\
\hline Sure shrink & 22.24 & 21.35 & 18.30 & 16.23 & 15.26 & 11.22 \\
\hline BMWT & 16.30 & 16.75 & 16.99 & 14.10 & 15.20 & 15.70 \\
\hline AMC-SSDA & 17.23 & 18.20 & 18.45 & 17.10 & 17.12 & 18.10 \\
\hline Proposed sequence & 26.92 & 30.60 & 34.12 & 26.69 & 30.37 & 33.90 \\
\hline
\end{tabular}

Mehran University Research Journal of Engineering \& Technology, Volume 37, No. 1, January, 2018 [p-ISSN: 0254-7821, e-ISSN: 2413-7219] 
method outperforms the existing approaches by clearly showing the morphological contents and enhancing the brightness. Results indicate that the traditional methods cannot preserve the structure, visual information, and brightness of the images and lose some important information from the images. Our method de-noises the images and preserves their visual and morphological information.Table 10 presents different quality values for MRI and CT scan images at standard deviations of 10 and 20. We use PSNR, CNR, UIQI, and SSIM as image quality evaluation metrics to evaluate the magnetic resonance images (Figs. 11-12). A high UIQI index indicates a high-quality image. SSIM method is used for similarity measurement between the two images. High SSIM, CNR, and PSNR indicate a high-quality image. A low DSSIM presents a high-quality image. From Table 10, it is clearly view that the proposed method performs efficiently when the standard deviation is increased. A carefully examination of the table reveal that our proposed approach perform well at S-dev 10 and AMC-SSDA, visu Shrink, sure Shrink and BMWT are perform near to our approach only in terms of UIQI, CNR and PSNR, but when the S-dev increases these approaches fail to provide good results in terms of objective evaluation, UIQI, PSNR, CNR, SSIM, and DSSIM.

TABLE 8. PSNR MEASURED VALUES AFTER ADDING GAUSSIAN NOISE AT DIFFERENT VARIANCE VALUES

\begin{tabular}{|c|c|c|c|c|c|c|c|c|c|}
\hline \multirow{3}{*}{ Different Resolution Images } & \multicolumn{3}{|c|}{ Variance 0.01} & \multicolumn{3}{|c|}{ Variance 0.05} & \multicolumn{3}{|c|}{ Variance 0.2} \\
\hline & \multicolumn{9}{|c|}{ Image Matrix Size } \\
\hline & $256 \times 256$ & $512 \times 512$ & $1024 \times 1024$ & $256 \times 256$ & $512 \times 512$ & $1024 \times 1024$ & $256 \times 256$ & $512 \times 512$ & $1024 \times 024$ \\
\hline Compared techniques & \multicolumn{9}{|c|}{ Values } \\
\hline DTCWPT & 28.37 & 31.81 & 35.28 & 27.83 & 31.31 & 34.73 & 26.76 & 30.32 & 33.63 \\
\hline Visu shrink & 27.23 & 27.16 & 29.96 & 23.83 & 24.89 & 22.64 & 21.23 & 21.79 & 19.33 \\
\hline Bayes shrink & 27.71 & 28.61 & 30.06 & 23.86 & 24.082 & 22.53 & 21.90 & 21.80 & 19.28 \\
\hline Sure shrink & 23.33 & 23.74 & 25.02 & 17.10 & 17.44 & 18.27 & 14.81 & 15.12 & 15.45 \\
\hline BMWT & 21.10 & 21.70 & 21.10 & 21.19 & 21.13 & 21.09 & 22.19 & 22.90 & 22.99 \\
\hline AMC-SSDA & 25.15 & 25.67 & 25.80 & 23.10 & 23.90 & 24.01 & 26.91 & 26.96 & 26.10 \\
\hline Proposed Sequence & 28.96 & 32.54 & 35.99 & 28.45 & 32.06 & 35.52 & 27.43 & 31.08 & 34.58 \\
\hline
\end{tabular}

TABLE 9. PSNR MEASURED VALUES AFTER ADDING SPECKLE NOISE AT DIFFERENT VARIANCE VALUES

\begin{tabular}{|c|c|c|c|c|c|c|c|}
\hline \multirow{2}{*}{ Different Resolution Images } & \multicolumn{5}{|c|}{ Variance 0.01} & \multicolumn{5}{|c|}{ Variance 0.5} \\
\cline { 2 - 8 } & \multicolumn{7}{|c|}{ Image Matrix Size } \\
\cline { 2 - 8 } & $256 \times 256$ & $512 \times 512$ & $1024 \times 1024$ & $256 \times 256$ & $512 \times 512$ & $1024 \times 1024$ \\
\hline Compared techniques & \multicolumn{7}{|c|}{ Values } \\
\hline DTCWPT & 25.54 & 29.19 & 32.33 & 25.04 & 28.70 & 31.80 \\
\hline Visu shrink & 28.88 & 29.58 & 34.45 & 26.93 & 27.096 & 27.85 \\
\hline Bayes shrink & 26.37 & 26.71 & 26.90 & 23.04 & 25.41 & 24.09 \\
\hline Sure shrink & 28.23 & 28.43 & 31.33 & 22.18 & 22.04 & 24.98 \\
\hline BMWT & 27.56 & 27.78 & 27.98 & 26.10 & 26.19 & 26.12 \\
\hline AMC-SSDA & 28.21 & 28.10 & 28.00 & 27.10 & 27.36 & 27.12 \\
\hline Proposed sequence & 29.52 & 29.94 & 33.50 & 25.75 & 29.45 & 33.50 \\
\hline
\end{tabular}

Mehran University Research Journal of Engineering \& Technology, Volume 37, No. 1, January, 2018 [p-ISSN: 0254-7821, e-ISSN: 2413-7219] 


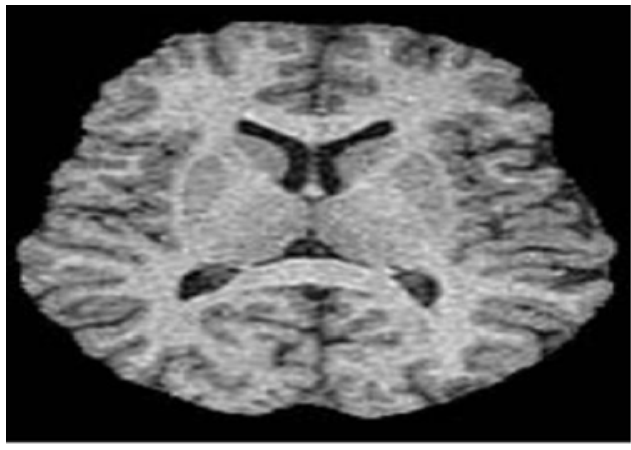

FIG. 11(a). ORIGINAL MAGNETIC RESONANCE IMAGE

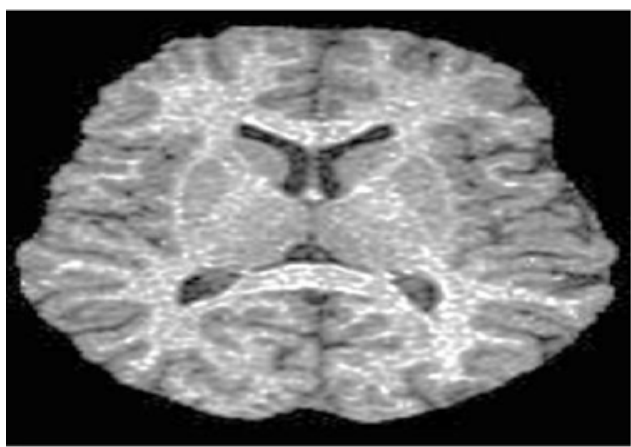

FIG. 11(b). IMAGE DE-NOISED WITH DTCWPT

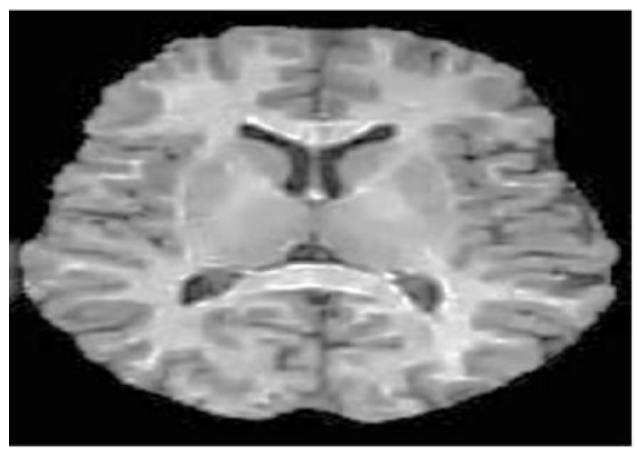

FIG. 11(c). IMAGE DE-NOISED WITH VISU SHRINK

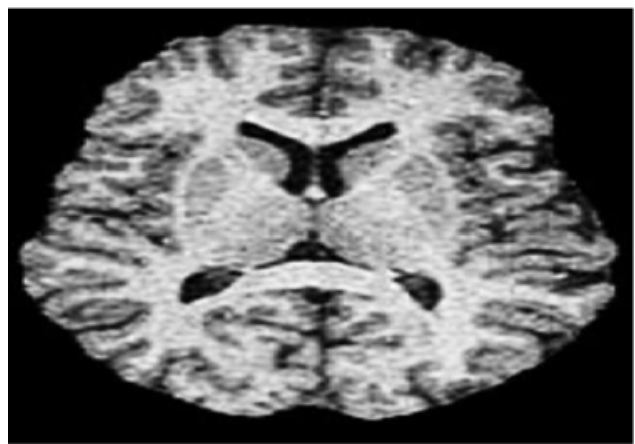

FIG. 11(d). IMAGE DE-NOISED WITH BAYES SHRINK

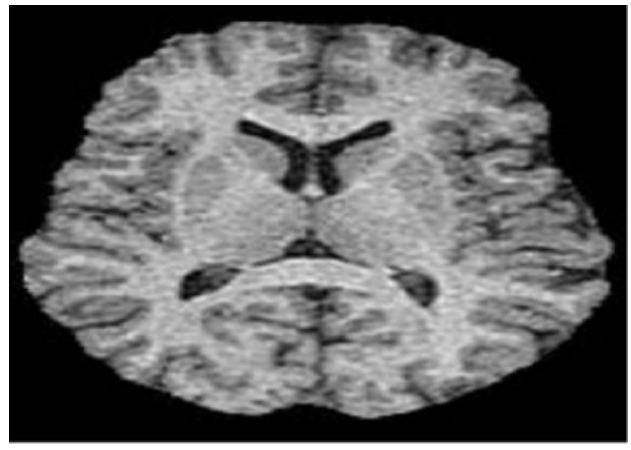

FIG. 11(e). IMAGE DE-NOISED WITH SURE SHRINK

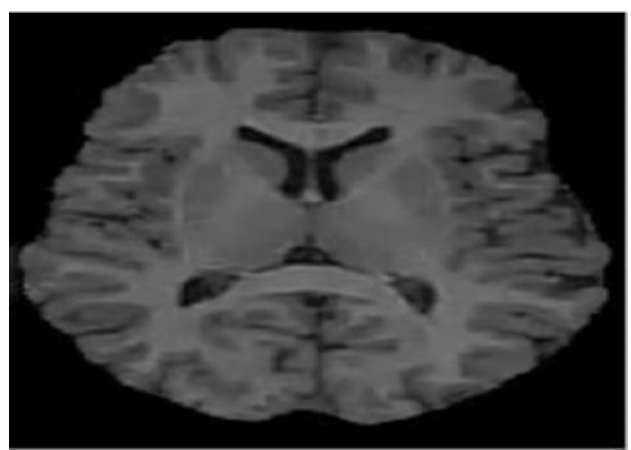

FIG. 11(f). IMAGE DE-NOISED WITH BMWT

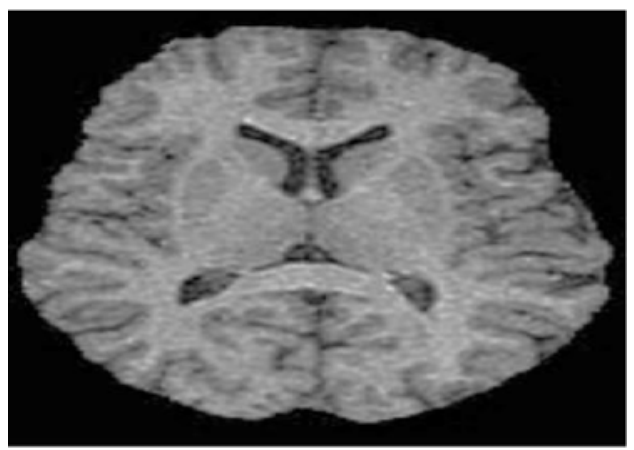

FIG. 11(g). IMAGE DE-NOISED WITH AMC-SSDA

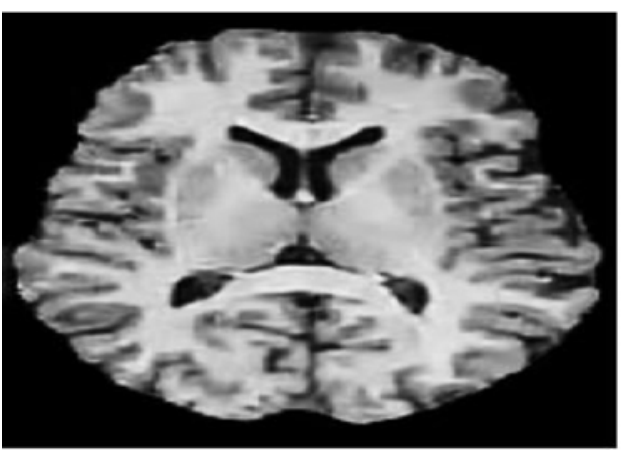

FIG. 11(h). IMAGE DE-NOISED WITH THE PROPOSED METHODS

Mehran University Research Journal of Engineering \& Technology, Volume 37, No. 1, January, 2018 [p-ISSN: 0254-7821, e-ISSN: 2413-7219] 


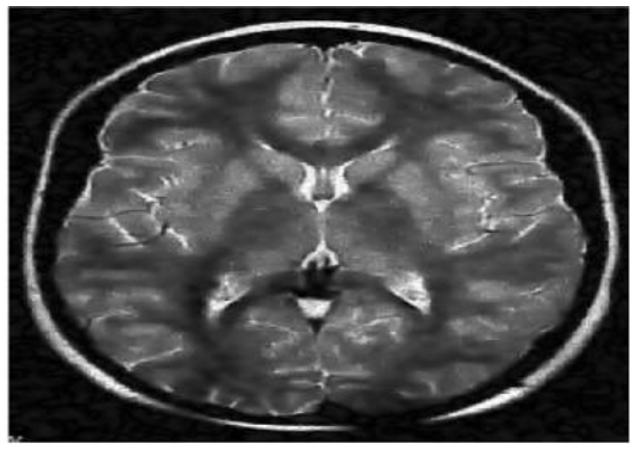

FIG. 12(a). ORIGINAL MAGNETIC RESONANCE IMAGE

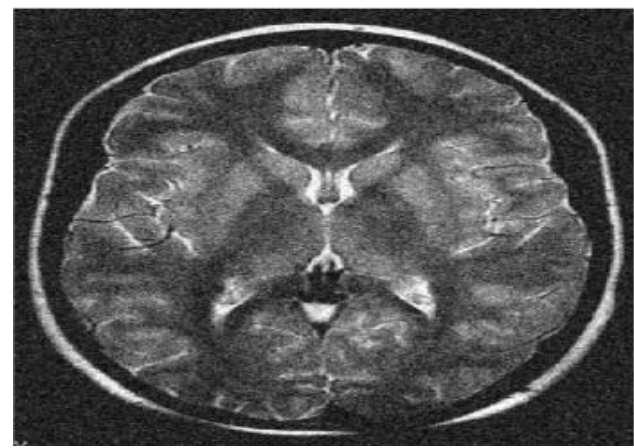

FIG. 12(b). IMAGE DE-NOISED WITH DTCWPT

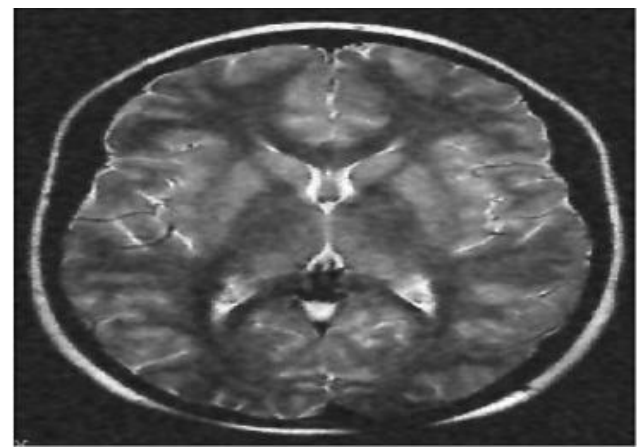

FIG. 12(c). IMAGE DE-NOISED WITH VISU SHRINK

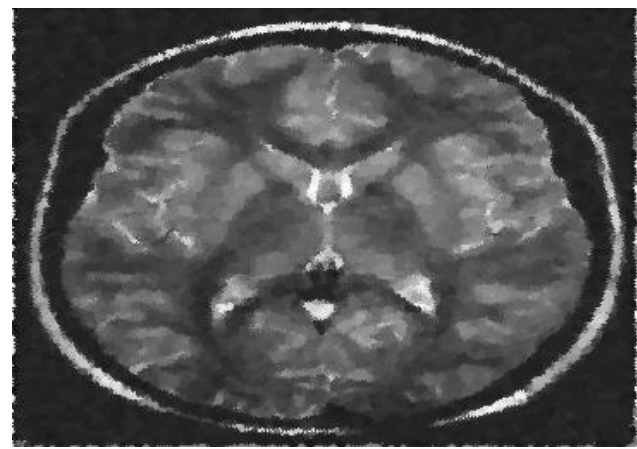

FIG. 12(d). IMAGE DE-NOISED WITH BAYES SHRINK

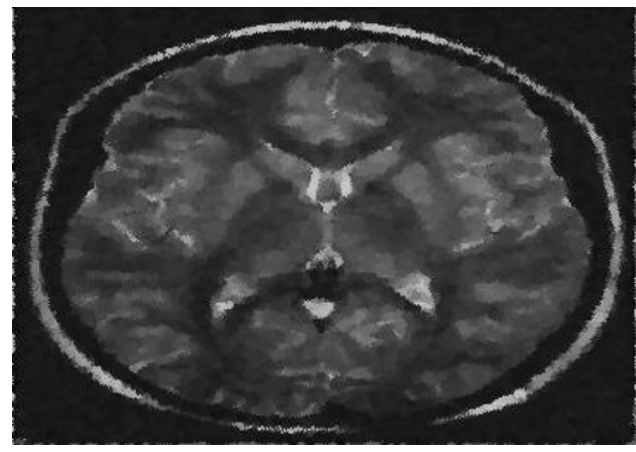

FIG. 12(e). IMAGE DE-NOISED WITH SURE SHRINK

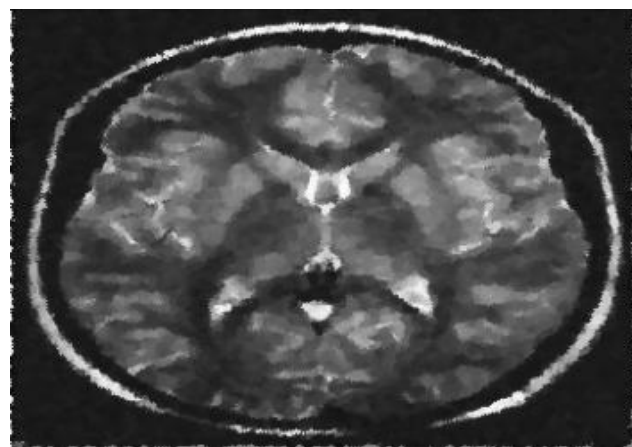

FIG. 12(f). IMAGE DE-NOISED WITH BMWT

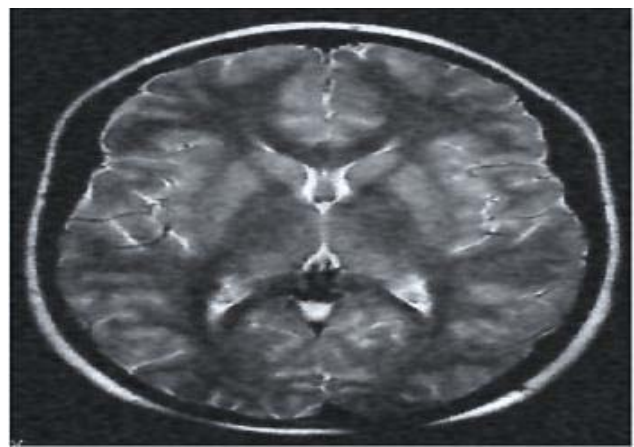

FIG. 12(g). IMAGE DE-NOISED WITH AMC-SSDA

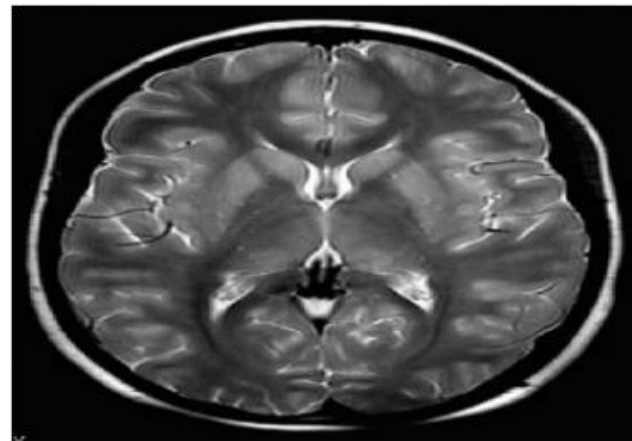

FIG. 12(h). IMAGE DE-NOISED WITH THE PROPOSED METHODS

Mehran University Research Journal of Engineering \& Technology, Volume 37, No. 1, January, 2018 [p-ISSN: 0254-7821, e-ISSN: 2413-7219] 
TABLE 10. PERFORMANCE OF DIFFERENT APPROACHES IN TERMS OF OBJECTIVE EVALUATION

\begin{tabular}{|c|c|c|c|c|c|c|c|c|c|}
\hline \multirow{2}{*}{ Image Type } & \multirow{2}{*}{$\begin{array}{l}\text { Standard } \\
\text { Deviation }\end{array}$} & \multirow{2}{*}{$\begin{array}{c}\text { Assessment } \\
\text { Parameters }\end{array}$} & \multicolumn{7}{|c|}{ De-Noising Methods } \\
\hline & & & DTCWPT & $\begin{array}{c}\text { Visu } \\
\text { Shrink }\end{array}$ & $\begin{array}{l}\text { Bayes } \\
\text { Shrink }\end{array}$ & $\begin{array}{c}\text { Sure } \\
\text { Shrink }\end{array}$ & BMWT & AMC-SSDA & $\begin{array}{l}\text { Proposed } \\
\text { Sequence }\end{array}$ \\
\hline \multirow{20}{*}{ MRI (Fig. 12) } & \multirow{5}{*}{10} & UIQI & 0.5680 & 0.8110 & 0.7921 & 0.8016 & 0.8710 & 0.8926 & 0.9109 \\
\hline & & PSNR & 28.21 & 28.32 & 31.33 & 29.50 & 32.33 & 30.43 & 34.58 \\
\hline & & CNR & 11.67 & 10.88 & 9.40 & 12.90 & 12.55 & 13.33 & 15.24 \\
\hline & & SSIM & 0.7590 & 0.7637 & 0.7722 & 0.7910 & 0.8111 & 0.7119 & 0.9597 \\
\hline & & DSSIM & 0.1205 & 0.1181 & 0.1139 & 0.1045 & .0944 & 0.1440 & 0.0201 \\
\hline & \multirow{5}{*}{20} & UIQI & 0.6680 & 0.7110 & 0.6921 & 0.7016 & 0.7710 & 0.7926 & 0.9309 \\
\hline & & PSNR & 30.65 & 30.99 & 33.44 & 32.41 & 33.69 & 31.79 & 35.48 \\
\hline & & CNR & 14.70 & 13.50 & 12.90 & 14.60 & 15.55 & 15.67 & 17.65 \\
\hline & & SSIM & 0.7190 & 0.7337 & 0.7522 & 0.7710 & 0.8011 & 0.7519 & 0.9697 \\
\hline & & DSSIM & .0905 & 0.1331 & 0.1239 & .1145 & 0.0994 & 0.1240 & 0.0151 \\
\hline & \multirow{5}{*}{10} & UIQI & 0.7680 & 0.6110 & 0.7121 & 0.8516 & 0.7610 & 0.8226 & 0.9409 \\
\hline & & PSNR & 29.39 & 29.90 & 29.16 & 30.98 & 31.90 & 31.10 & 33.90 \\
\hline & & CNR & 14.90 & 14.10 & 13.80 & 13.85 & 14.01 & 14.60 & 16.20 \\
\hline & & SSIM & 0.7686 & 0.7988 & 0.8080 & 0.8012 & 0.8211 & 0.7519 & 0.9417 \\
\hline & & DSSIM & 0.1157 & 0.1006 & .0960 & 0.0994 & .0894 & 0.1240 & 0.0291 \\
\hline & \multirow{5}{*}{20} & UIQI & 0.6780 & 0.6110 & 0.7221 & 0.7316 & 0.8410 & 0.8226 & 0.9209 \\
\hline & & PSNR & 31.45 & 30.33 & 30.90 & 32.10 & 34.15 & 33.12 & 36.34 \\
\hline & & CNR & 14.23 & 15.10 & 15.60 & 15.98 & 16.20 & 16.55 & 18.45 \\
\hline & & SSIM & 0.7486 & 0.7688 & 0.7680 & 0.7512 & 0.7911 & 0.7829 & 0.9317 \\
\hline & & DSSIM & 0.1257 & 0.1156 & 0.1160 & 0.1244 & 0.1044 & 0.1085 & 0.03415 \\
\hline
\end{tabular}

\section{CONCLUSION}

In this paper, we propose a technique in combination of curvelet and ridgelettransforms along with CS. Curvelet and ridgelet transforms have the same reconstructions properties, give a stables restoration underneath distresses of image coefficient, and are organized in training.We split the nonstandards frequencies subband arisings in theoreticals actions of curvelet into two standard sdyadics frequencies sub-bands, and we manage them independently. Through this process, we will find good results. Thecurvelet and ridgelet transforms are well modified to decompositions of subbands. For instance, a substitutive plan using decimated
2D wavelet transforms familiarizes visual artifacts near the strong edges.

CS algorithm is used for to optimize curvelet coefficients. Our new hybrid method combines curvelet transform along with ridgelet transform with an optimization technique named as CS. The main function of CS is to optimize and maintain the main feature and morphological structure of an image without loss of information. The proposed approach uses the CS algorithm with curvelet transform. Results of proposed technique are compared with those of other four approaches, i.e.Visu shrink, Bayes shrink, Sure shrink, and versatile wavelet transform. Our method outperform the other traditional approaches. 


\section{ACKNOWLEDGMENT}

Authors would like to acknowledge with thanks the anonymous referees for their useful suggestions that led us to enhance the quality of the paper. Authors are also thankful to the International Islamic University, COMSATS Institute of Information Technology, Islamabad and University of Gujrat, Pakistan, for providing platform to carry out this research.

\section{REFERENCES}

[1] Sui, J., Pearlson, G., Caprihan, A., Adali, T., Kiehl, K.A., Liu, J., Yamamoto, J., and Calhoun, V.D., "Discriminating Schizophrenia and Bipolar Disorder by Fusing FMRI and DTI in a Multimodal CCA+ Joint ICA Model”, Neuroimage, Volume 57, No 3, pp. 839-855, 2011.

[2] Donoho, D.L., and Johnstone, I.M., "Adapting to Unknown Smoothness via Wavelet Shrinkage”, Journal of the American Statistical Association, Volume 90, No. 432, pp. 1200-1224, 1995.

[3] Donoho, D.L., "De-Noising by Soft-Thresholding”, IEEE Transactions on Information Theory, Volume 41, No. 3,pp. 613-627, 1995.

[4] Chang, S.G., Yu, B., and Vetterli, M., "Adaptive Wavelet Thresholding for Image Denoising and Compression”, IEEE Transactions on Image Processing,Volume 9, No. 9, pp. 1532-1546, 2000

Al-Sbou, Y.A., “Artificial Neural Networks Evaluation as an Image Denoising Tool”, World Applied Science Journal, Volume 17, pp. 218-227,2012.

Ilango, G., andMarudhachalam, R., "New Hybrid Filtering Techniques for Removal of Gaussian Noise from Medical Images”, Journal of Engineering \& Applied Sciences, Volume 6, No. 2, pp. 1-8, 2011.

Pizurica, A., “A Versatile Wavelet Domain Noise Filtration Technique for Medical Imaging”, IEEE Transactions on Medical Imaging,Volume 22, No. 3, pp. 323-331, 2003.

[8] Jin, Y., “Multiscale Denoising and Enhancement of 3DRotational X-Ray Imaging for Percutaneous Vertebroplasty”, Proceedings of IEEE 25th Annual International Conferenceon Engineering in Medicine and Biology Society, 2003.
[9] Anand, C.S., and Sahambi, J.,’MRI Denoising Using Bilateral Filter in Redundant Wavelet Domain”, IEEE Region Conference on TENCON, 2008.

[10] Yan, X., "Noise Removal of MRI Data with Edge Enhancing”, IEEE 5th International Conference on Bioinformatics and Biomedical Engineering,2011.

[11] Tayel, M.B., Abdou, M.A., and Elbagoury, A.M., “An Efficient Thresholding Neural Network Technique for High Noise Densities Environments”, International Journal of Image Processing, Volume 5, No. 4, pp. 403, 2011.

[12] Velayudham, A., Kanthavel, R., and Kumar, K.M., "A Novel and Hybrid Optimization Mechanism for Denoising and Classification of Medical Images Using DTCWPT And Neuro-Fuzzy Classifiers”, International Review on Computers and Software,Volume 9, No. 3, p. 513-525, 2014.

[13] Van De Ville, D., "Noise Reduction by Fuzzy Image Filtering”, IEEE Transactions on Fuzzy Systems, Volume 11, No. 4, pp. 429-436, 2003.

[14] Chitroub, S., "Principal Component Analysis by Neural Network. Application: Remote Sensing Images Compression and Enhancement”, Proceedings of 10th IEEE International Conference on Electronics, Circuits and Systems, 2003.

[15] Vetrivelan, P., and Kandaswamy, A., "Medical Image Denoising Using Wavelet-Based Ridgelet Transform”, Emerging Research in Electronics, Computer Science and Technology, Springer, Volume 12, pp. 235-243, 2014.

[16] Pragada, S., and Sivaswamy, J., "Image Denoising Using Matched Biorthogonal Wavelets”, $6^{\text {th }}$ IEEE Indian Conference on Computer Vision, Graphics \& Image Processing, 2008.

[17] Benazza-Benyahia, A., and Pesquet, J., "An Extended Sure Approach for Multicomponent Image Denoising”, Proceedings of IEEE International Conference on Acoustics, Speech, and Signal Processing, 2004.

[18] Jalobeanu, A., "Satellite and Aerial Image Deconvolution Using an EM Method with Complex Wavelets”, Proceedings of IEEE International Conference on Image Processing, 2002. 
[19] Prakash, O., and Khare, A., "Medical Image Denoising Based on Soft Thresholding Using Biorthogonal Multiscale Wavelet Transform”, International Journal of Image and Graphics, Volume14, pp. 12-18, 2014.

[20] Huang, X., Madoc, A., and Cheetham, A.,’Multi-Noise Removal from Images by Wavelet-Based Bayesian Estimator”, Proceedings of IEEE $6^{\text {th }}$ International Symposium on Multimedia Software Engineering, 2004.

[21] Huang, X, Madoc, A.C., and Cheetham, A.D., "WaveletBased Bayesian Estimator for Poisson Noise Removal from Images”, Proceedings. of IEEE International Conference on Multimedia and Expo, 2003.

[22] Chen, C., and Wang. X.,”A Novel Theory of SAR Image Restoration and Enhancement with ICA", Proceedings of IEEE International Symposium on Geoscience and Remote Sensing, 2004.

[23] Zhang, C., and Xiaoqin, L.,’Typhoon Image Denoising in Curvelet Domain”, $2^{\text {nd }}$ IEEE International Conference on Bio-Inspired Computing: Theories and Applications, 2007.

[24] Rashedi, E., and Zarezadeh, A.,"Noise Filtering in Ultrasound Images Using Gravitational Search Algorithm”, Iranian IEEE Conference on Intelligent Systems, 2014

[25] Qing-Hang, H., “The Processing of the Degraded Medical Digital Image's Image Enhancement”, Proceedings of IEEE Annual International Conference on Engineering in Medicine and Biology Society, 2004.

[26] Tian, H., "Improved Partial Differential Equation-Based Method to Remove Noise in Image Enhancement", 2011.

[27] Krissian, K., and Aja-Fernández, S., "Noise-Driven Anisotropic Diffusion Filtering of MRI”, IEEE Transactions on Image Processing, Volume 18, No. 10, pp. 2265-2274,2009.

[28] Ahmed, S.S., "Bilateral Filtering and Wavelets based Image Denoising: Application to Electron Microscopy Images with Low Electron Dose”, International Journal on Recent Trends in Engineering and Technology, Volume 11, No. 1, pp. 1-6, 2014.

[29] Dixit, A., and Sharma, P., "A Comparative Study of Wavelet Thresholding for Image Denoising”, 2014.
[30] Kumar R.V.V., and Kumar, M.P., "Denoising of MRI and X-Ray Images Using Dual Tree Complex Wavelet and Curvelet Transforms”,IEEE International Conference on Communications and Signal Processing, 2014.

[31] Candes, E.J., and Donoho, D.L, “Curvelets: A Surprisingly Effective Nonadaptive Representation for Objects with Edges”, DTIC Document,2000.

[32] Candès, E.J., and Donoho, D.L., "Ridgelets: A Key to Higher-Dimensional Intermittency? Philosophical Transactions of the Royal Society of London”, SeriesA: Mathematical, Physical and Engineering Sciences, Volume 357, No. 1760, pp. 2495-2509,1999.

[33] Candes, E., "Fast Discrete Curvelet Transforms", Multiscale Modeling \& Simulation, Volume 5, No. 3, pp. 861-899,2006.

[34] Donoho, D.L., and Duncan, M.R., “Digital Curvelet Transform: Strategy, Implementation, and Experiments”, AeroSense, International Society for Optics and Photonics,2000.

[35] Deans, S., "The Radon Transform and Some of Its Applications”, Florida: Krieger Publishing Company, 1983.

[36] Yang, X.-S., and Deb, S.,”Cuckoo Search via Lévy Flights”, IEEE World Congress on Nature \& Biologically Inspired Computing, 2009

[37] Yildiz, A.R., "Cuckoo Search Algorithm for the Selection of Optimal Machining Parameters in Milling Operations”, The International Journal of Advanced Manufacturing Technology,. Volume 64, No 4, pp. 55-61,2013

[38] Sharma, D., and Singh, M., "A Comparitive Analysis of Thresholding Techniques Used in Image Densing through Wavelets”, 2008.

[39] Wang, Z., “Image Quality Assessment: From Error Visibility to Structural Similarity”, IEEE Transactions on Image Processing, Volume 13, No. 4, pp. 600-612, 2004.

[40] Bouzerdoum, A.A., Havstad, A., and Beghdadi, A.,'Image Quality Assessment using a Neural Network Approach”, Proceedings of $4^{\text {th }}$ IEEE International Symposium on Signal Processing and Information Technology, 2004. 